Highly Energetic Physical Processes and

Mechanisms for Emission from Astrophysical Plasmas

IAU Symposium, Vol. 195, 2000

P. C. H. Martens, S. Tsuruta, and M. A. Weber, eds.

\title{
X-ray Emission Characteristics of Pulsars
}

\author{
W. Becker \\ Max-Planck-Institut für extraterrestrische Physik, Giessenbachstrasse 1, \\ Postfach 1603, 85740 Garching, Germany
}

\begin{abstract}
Recent X-ray observatories such as ROSAT, ASCA, RXTE, BeppoSAX, and Chandra have achieved important progress in neutron star and pulsar astronomy. The identification of Geminga as a rotationpowered pulsar, the discovery of X-ray emission from millisecond pulsars, and the identification of cooling neutron stars are only a few of the fascinating results. In the following, I will give a brief review on the X-ray emission properties of rotation-powered pulsars and their wind nebulae.
\end{abstract}

\section{Introduction}

Neutron stars are among the most fascinating astronomical objects in the universe. Born in the imploding core of a supernova, they provide a unique class of stellar objects with properties that make them nearly ideal probes for investigating a wide variety of physical problems.

A primary goal in the study of rotation-powered pulsars at X-ray energies is to search for thermal X-ray emission from cooling neutron stars (Ögelman 1995; Tsuruta 1998; Pavlov 2000, these Proceedings). The close link between the thermal evolution of neutron stars and the physical characteristics of neutron star material at supernuclear densities provides an important clue to the empirical study of matter at extreme energies and supernuclear densities. Comparing measured neutron star temperatures with the theoretical predictions based on different equations of state thus provides the empirical basis essential for the verification of neutron star models and cooling theories.

Another point of interest is the physical mechanism operating in the pulsar magnetosphere to produce the intense, broadband-beamed radiation. It is well known that the radio emission of pulsars is due to coherent processes, and coherent curvature radiation has been identified as the most promising mechanism (see Michel 1991 and references therein). On the other hand, the optical, X-ray, and gamma-ray emission observed in pulsars must be incoherent. Therefore, the fluxes in these energy bands are directly proportional to the densities of the radiating high-energy electrons in the acceleration regions, no matter what radiation process (synchrotron radiation, cyclotron radiation, or inverse Compton effects) is at work. High-energy observations of neutron stars thus provide the key for an understanding of the pulsars' emission mechanisms.

Before the launch of ROSAT, not much was known about the X-ray emission properties of rotation-powered pulsars. Pulsed X-rays had been detected only from the three youngest and most powerful pulsars (Crab, PSR 1509-58, 
and PSR 0540-69), and fluxes were known for only 4 more pulsars (Seward \& Wang 1988). Theoretical models explaining pulsar emission mechanisms and high-energy radiation characteristics were strongly biased by the special results found for these few young pulsars, forming a very limited sample of objects not representative of the whole group of rotation-powered pulsars. This situation has changed in the last years. Studying neutron stars with the X-ray satellites launched in the early 1990s has increased the number of X-ray detected pulsars to $35^{1}$ by now (Becker \& Trümper 1997). Although this is still less than $5 \%$ of the known radio-pulsar population, the detected objects cover a wide range of ages, magnetic field strengths, and spin periods, and represent a statistically unbiased sample in terms of their stellar parameters.

\section{Emission Properties}

To get into the X-ray emission properties of the detected pulsars, we group the whole sample into five classes according to their spin-down age $\tau=P / 2 \dot{P}$.

\subsection{The Crab-like Pulsars}

It is well established that in the young rotation-powered pulsars with ages $\leq$ 2000 years magnetospheric emission dominates. In the case of the Crab pulsar, for example, at least $\sim 75 \%$ of the total soft $\mathrm{X}$-ray flux is magnetospheric emission characterized by a power-law spectrum and sharp pulses. Thermal cooling radiation from the Crab pulsar surface is buried by the intense emission from the pulsar's synchrotron nebula. An upper limit of the unpulsed flux within the $0.1-2.4 \mathrm{keV}$ range, deduced from the DC level of the soft X-ray pulse profile, was taken by Becker \& Aschenbach (1995) as an upper limit for the thermal flux from the young neutron star. Knowing the Crab pulsar's surface temperature allows a comparison with theoretical predications at an early time in the neutron star's thermal evolution. A temperature of less than $2 \times 10^{6} \mathrm{~K}(3 \sigma)$ was found to be marginally consistent with standard cooling models.

The ROSAT HRI data taken from the Crab nebula have been used to improve our understanding of this object in many aspects. In a long term study of the Crab nebula using ROSAT HRI data, spanning a period of more than 6 years, Greiveldinger \& Aschenbach (1999) have shown that the X-ray intensity of the synchrotron nebula varies on time scales of years by about $20 \%$. The intensity variations are found to be confined to rather large $\left(\sim 25^{\prime \prime} \times 25^{\prime \prime}\right)$ and well-defined regions in the torus and may point towards a decrease in the relativistic wind's bulk motion. Using the instruments aboard Chandra, it will be easy to further investigate these long-term variations. The first images taken with the HETG aboard Chandra provided already spectacular details on the nebula structure, its torus, and the jet-like feature associated with the pulsarwind outflow. To demonstrate the recent progress in X-ray astronomy, images from the Crab pulsar and plerion as seen by Chandra's HETG and the ROSAT HRI are shown in Figure 1.

\footnotetext{
${ }^{1}$ For a tabulated summary of the X-ray detected rotation-powered pulsars, their X-ray luminosities, and parameters, see http://ww.xray.mpe.mpg.de/ web/bt97_update.html.
} 


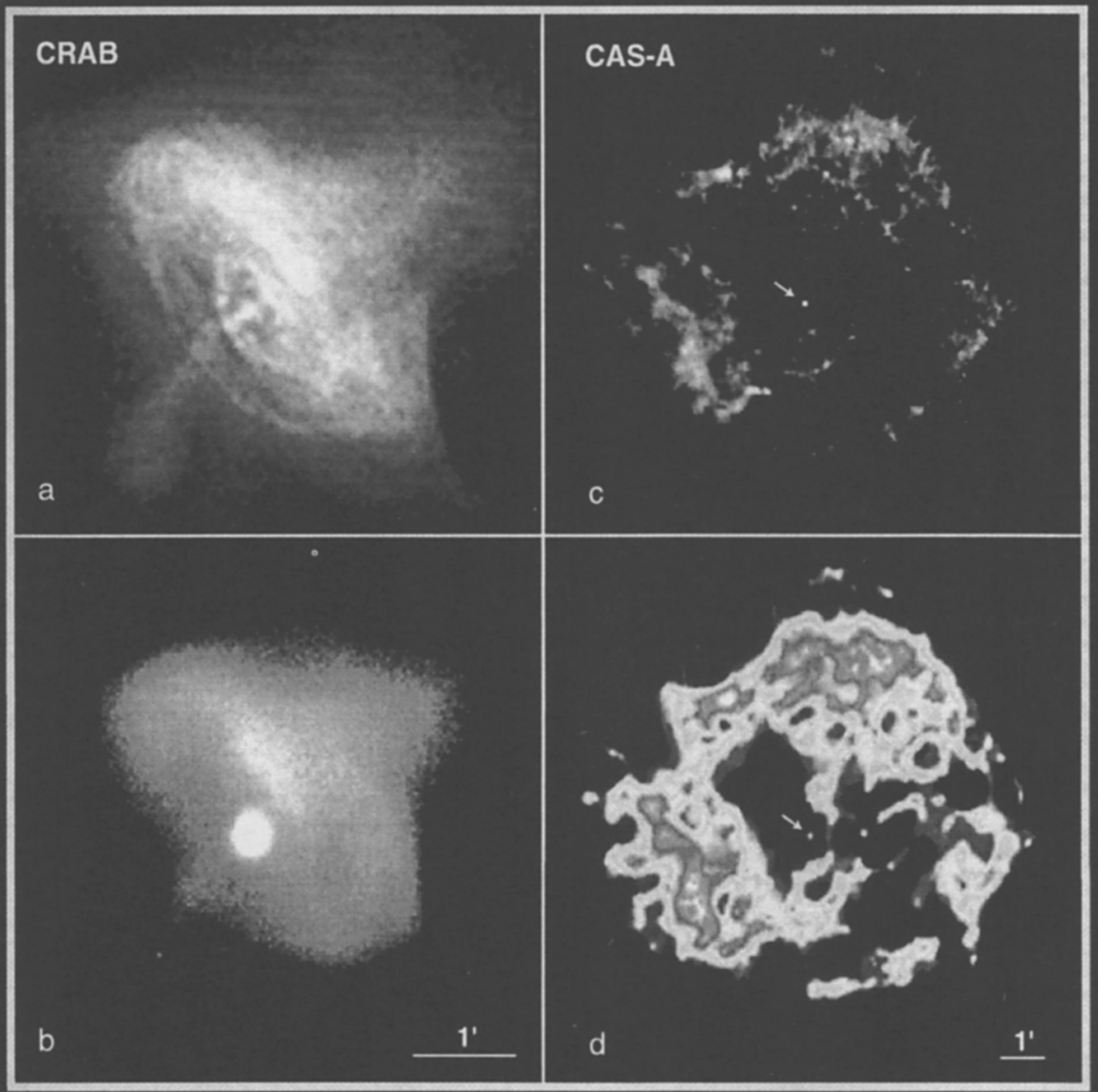

Figure 1. The Crab as observed with the HETG aboard Chandra (a) and the ROSAT HRI (b). Image (c) shows another recent discovery made by Chandra: the point source close to the geometrical center of Cas-A, a 320-year young supernova remnant. The corresponding ROSAT HRI image is shown in (d). Although the central point source was already detected in deep ROSAT HRI observations, only the unprecedented high spatial resolution provided by Chandra allowed the identification of the emission as being from a young neutron star or a black hole. Spectral analysis using the Chandra ACIS data shows that a power-law (nonthermal) spectrum implies a slope which is steeper, and a luminosity which is lower, than those found in other young (i.e., Crab-like) pulsars. Spectral fits using thermal spectra result in a higher temperature and smaller emitting area than expected for cooling radiation from a hot, young neutron star. Assuming that the point source is a young neutron star and that the emission is thermal and comes from the whole surface, an upper limit on the surface temperature is $\sim 2.5 \times 10^{6} \mathrm{~K}$. A search for radio emission from the point source at $21 \mathrm{~cm}$ using the 100 -meter dish at Effelsberg did not detect pulsed radio emission from the position of the X-ray point source. The problem in searching for faint, pulsed radio emission from a young pulsar in Cas- $A$ is that the supernova remnant itself is a very bright radio source which can easily bury any faint emission from a young pulsar. 
Similar emission properties as found for the Crab are observed from PSR 1509-58 in the supernova remnant MSH 15-52. The pulsed X-ray emission in the ROSAT band is found to have a pulsed fraction of $65 \pm 4 \%$ (Becker \& Trümper 1997) and a soft X-ray pulse which is aligned with the hard pulses detected at $20-170 \mathrm{keV}$ by BATSE and OSSE (Ulmer et al. 1994). Based on ASCA observations, Tamura et al. (1996) found evidence for the existence of a jet-like structure extending from the pulsar toward the second region of enhanced X-ray emission, RCW 89. The authors proposed that the jet-like structure is a stream of relativistic particles (i.e., pulsar-wind outflow) from PSR 1509-58 which is colliding with the ambient interstellar medium and/or supernova ejecta to form the X-ray emission associated with RCW 89. They have also shown that while PSR 1509-58 and its surrounding synchrotron nebula have a hard power-law spectral shape, the spectrum from RCW 89 has a prominent soft component with at least two lines at $0.9 \mathrm{keV}(\mathrm{Ne} \mathrm{K} \alpha)$ and $1.3 \mathrm{keV}(\mathrm{Mg} \mathrm{K} \alpha)$. However, the limited angular resolution of ASCA did not allow the pulsar to be spatially separated from the synchrotron nebula, nor to resolve RCW 89 with any fine structures seen in the optical or radio bands. This became possible with the ROSAT HRI, which resolved the RCW 89 region into a partial ring of tight knots that closely follow the radio morphology of the region. Brazier \& Becker (1997) therefore have proposed that the appearance of the synchrotron nebula surrounding PSR 1509-58 is the result of a torus and a jet, similar to what is found in the Crab pulsar synchrotron nebula and for Vela. The RCW 89 region is a region where the collimated pulsar wind is impacting the outer shell of the supernova remnant.

\subsection{The Vela-type Pulsars}

Different from the Crab-like pulsars are rotation-powered pulsars in the age bracket $\sim 10^{4}-10^{5}$ years (e.g., the Vela-pulsar, PSR B1706-44, B1046-58, B180021, B1823-13, B1951+32, and J1105-6107). They exhibit strong, steady emission from a pulsar-powered synchrotron nebula (mostly unresolved), combined with a small pulsed contribution of magnetospheric or thermal origin dominating the emission in the range $\sim 0.1-0.5 \mathrm{keV}$ (Becker \& Trümper 1996). X-ray pulses are only detected for the Vela pulsar (Ögelman, Finley, \& Zimmerman 1993). The other Vela-type pulsars are more distant and suffer from photoelectric absorption which prevents the detection of their soft pulses in the presence of the dominant nebula emission. This interpretation is also true for PSR 1951+32 in CTB 80. A report of a $\sim 2 \sigma$ signal by Safi-Harb, Ögelman, \& Finley (1995) could not be established in a deep ROSAT HRI observation nor, more recently, by observations using the Rossi Timing Explorer. The pulsed fraction upper limit deduced from the ROSAT HRI data is about $9 \%$.

Apart from the Vela pulsar itself, there is no spectral information for these sources below $0.5 \mathrm{keV}$, which means that their blackbody component is invisible because of photoelectric absorption. The spatial structure of the Vela pulsar/nebula has been resolved recently with the ROSAT HRI (Marquart \& Ögelman 1999). Figure 2 shows the Vela pulsar and plerion as seen in a deep HRI observation. 


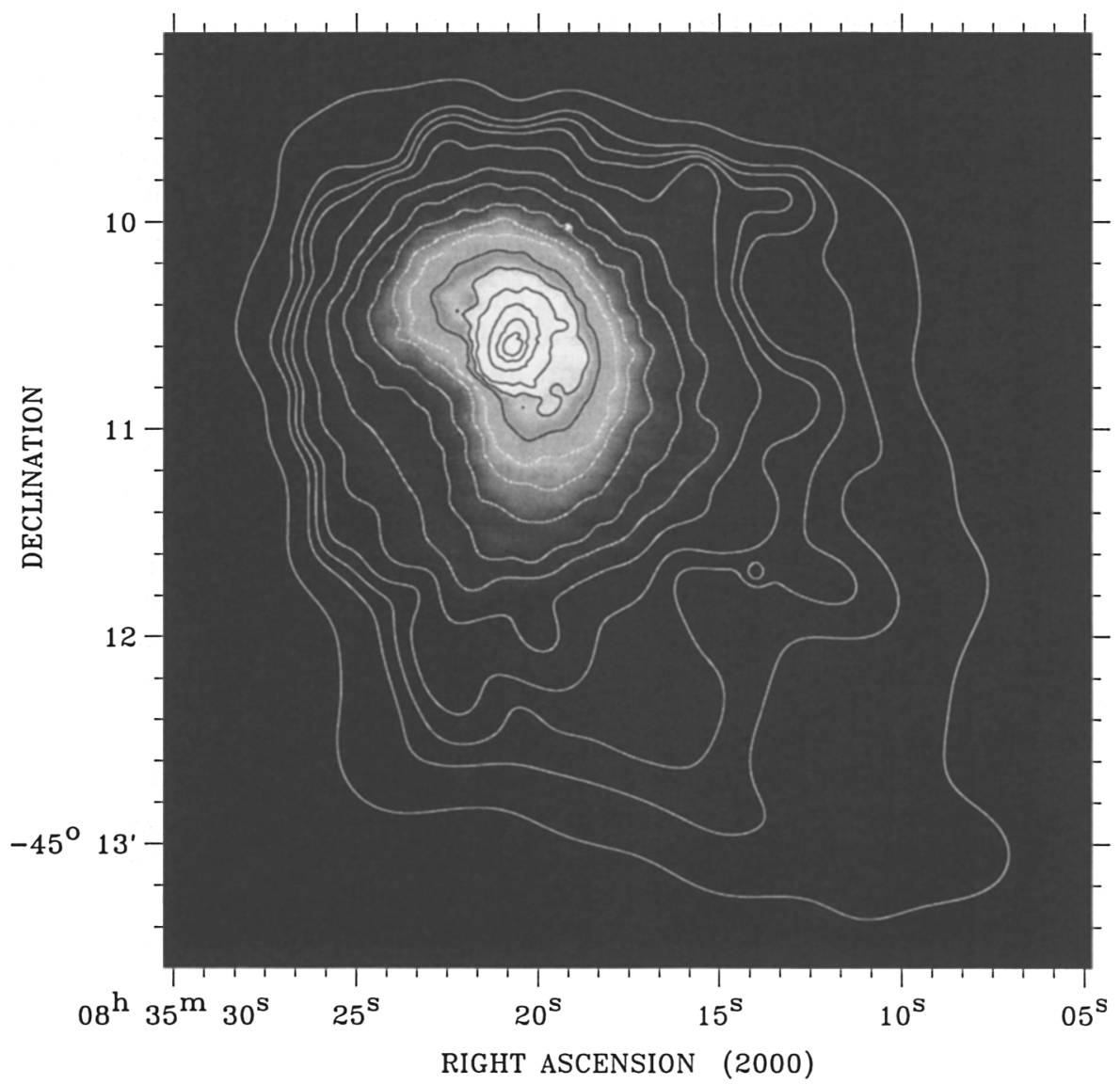

Figure 2. Vela pulsar and plerion as observed with the ROSAT HRI. All HRI data taken between May 1994 and June 1996 (corresponding to a total exposure time of $210 \mathrm{ksec}$ ) have been merged together to produce the image. The structure, shape, and size of the nebula are different from what is observed from the Crab and PSR 1509-58. The nebula luminosity is $0.04 \%(0.1-2.4 \mathrm{keV})$ of the pulsar's spin-down energy, whereas, for the Crab nebula. it is $\sim 5 \%$, and, for the nebula around PSR 1509-58, it is $\sim 1 \%$ (in the same energy band). Also, the soft X-ray spectrum of the Vela pulsar is different from that of Crab-like pulsars. While, for the latter two, the photon-index of the pulsar emission is $\alpha \sim 1.5$, it is $\alpha \sim 2.7$ for the Vela. The nebula spectrum of all plerions seen around young pulsars is well fitted by a power-law with photon-index $\alpha=2$. 


\subsection{The Cooling Neutron Stars}

These are the three middle-aged pulsars: Geminga, PSR 0656+14, and 105552. The soft X-ray emission properties of these pulsars are characterized by a dichotomy, i.e., the spectra are best described by a two-component model in which the soft emission is represented by a blackbody spectrum and the hard component either by a thermal spectrum or by a power-law. The existence of two spectral components is also confirmed by phase-resolved analysis. All three pulsars show a phase shift of $\sim 100^{\circ}$ and a change in the pulsed fraction from $\sim$ $10-30 \%$ below a transition point of $0.5-0.6 \mathrm{keV}$, rising up to $\sim 20-65 \%$ above. The X-ray pulse profiles for both the soft and the hard components are found to be sinusoidal.

The soft thermal emission is assigned to be cooling emission from the neutron star's surface. The modulation of this emission can be explained by nonuniformities in the surface temperature due to the presence of a strong magnetic field which gives rise to an anisotropic heat flow in the neutron star's outer layers. The radius of the emitting area obtained by using $R_{b b}=d / T^{2} \sqrt{f_{b o l} / \sigma}$, in which $f_{b o l}$ and $T$ denote the fitted bolometric flux and temperature of the pulsar's soft component and $\sigma$ the Stefan-Boltzmann constant, are found to be in the range $\sim 7-30 \mathrm{~km}$.

Based on ROSAT data, the hard spectral components of these pulsars can be interpreted as magnetospheric emission or thermal radiation from polar hot spots. Because of bandwidth limitations, it is not possible to discriminate between these possibilities, but ASCA observations have shown recently that the hard component of Geminga is characterized by a power-law, implying a magnetospheric origin (Halpern \& Wang 1997). Similar results have been reported for 1055-52 (Wang et al. 1998) and PSR 0656+14 (Greiveldinger et al. 1996), although, in the latter case, the limited photon statistics prevent a clear distinction between a thermal and nonthermal origin of the harder emission.

The existence of X-ray-bright pulsar-wind nebula as a common feature surrounding rotation-powered pulsars was recently reported on the basis of ASCA observations (Kawai \& Tamura 1996). Indeed, the ASCA data taken from PSR 0656+14, 1055-52, and Geminga (as well as from other pulsars) show a faint, diffuse, and somewhat clumpy emission around these pulsars, interpreted as a result of an interaction of a pulsar wind outflow with the surrounding interstellar matter (Kawai \& Tamura 1996; Shibata et al. 1997). The ROSAT (and if available the BeppoSAX) data of these sources were re-analyzed recently by Becker et al. (1999) with the aim to search for faint point sources which-due to the wide ASCA point-spread function of about 3 arcmin (FWHM) - could result in such a pattern of diffuse emission and knots in the ASCA data. At least for Geminga, PSR 0656+14, and PSR 1055-52, faint X-ray counterparts were found in the ROSAT data which correspond well with the diffuse structure and knots seen in the ASCA data. Becker et al. (1999) therefore conclude that the X-ray knots seen around these pulsars by Kawai \& Tamura (1996) are unlikely to be associated with the pulsars and are unrelated background objects.

\subsection{Pulsars Old and Close in Space}

Besides the young pulsars and those whose surface cooling is visible in the $\mathrm{X}$-ray wave band, X-ray emission is detected from three other pulsars: PSR B1929+10, 
B0950+08, and B0823+26. All are characterized by a spin-down age of $\sim 0.2-$ $3 \times 10^{7}$ years and a close distance of $\sim 0.12-0.38 \mathrm{kpc}$. Pulsed X-ray emission, however, could only be detected from PSR B1929+10 (Yancopoulos, Hamilton, $\&$ Helfand 1994). Its X-ray pulse profile is very broad with a single pulse stretching across almost the entire phase cycle. Spectral information is only available for B1929+10. Its soft X-ray spectrum can be equally well fitted with a powerlaw of photon-index $\sim 2$ and a blackbody spectrum. A faint synchrotron tail which is oriented along the pulsar's proper motion direction was found in the ROSAT PSPC data by Wang, Li, \& Begelman (1993).

\subsection{The Millisecond Pulsars}

Millisecond pulsars form a separate group among the rotation-powered pulsars. They are distinguished by their small spin periods $(P \leq 20 \mathrm{~ms})$ and high rotational stability $\left(\mathrm{d} P / \mathrm{d} t \approx 10^{-18}-10^{-21}\right)$ and, consequently, they are very old objects with spin-down ages of typically $10^{9}-10^{10}$ years and magnetic field strengths of the order of $10^{8}-10^{10} \mathrm{G}$.

ROSAT, with a significantly higher sensitivity compared to previous X-ray satellites, allowed, for the first time, the detection of X-ray emission from objects as faint as millisecond pulsars. However, although eleven of the 35 detected rotation-powered pulsars belong to the small group of millisecond pulsars, the origin of the detected X-ray emission for most of them is not yet known ${ }^{2}$. Six of the eleven ms-pulsars (PSR B1957+20, J1012+5307, B0751+18, J1744-1134, $\mathrm{J} 1024-0719$, and J0030+0451) are identified in X-rays only by their positional coincidence with the radio pulsar and, in view of the low number of detected counts, do not provide much more than a rough flux estimate. These objects are so faint that the sensitivity of Chandra and XMM is needed to detect enough photons for a detailed spectral and temporal study in the soft and hard band beyond $2 \mathrm{keV}$. More detailed results are found for the other five ms-pulsars which all provide important empirical information on the pulsar's X-ray emission mechanisms.

- The 3-ms pulsar PSR 1821-24 in the globular cluster M28 shows Crablike X-ray pulses up to $\sim 17 \mathrm{keV}$ (Saito et al. 1997; Kawai \& Saito 1999). The two sharp peaks in the pulse profile and the power-law nature of the spectrum undoubtedly argue for a magnetospheric (nonthermal) origin of the detected emission. The alignment between a radio and X-ray pulse component adds further support to this interpretation and implies a common emission site for the main X-ray and radio pulse components observed at $800 \mathrm{MHz}$. The photon-index for the pulsed emission is found to be $\alpha \sim 1.2$, using RXTE (Kawai \& Saito 1999). Imaging M28 with the ROSAT HRI has shown two separate sources: a point source (RX J1824.52452P) consistent with the ms-pulsar position and a brighter but extended source RX J1824.5-2452E, whose nature is not yet clear. The spectral results obtained from ASCA for RX J1824.5-2452Efavor the model in which

\footnotetext{
${ }^{2} \mathrm{~A}$ recent review on the X-ray emission properties of millisecond pulsars, which summarizes the current emission properties and observational status in a much wider and more complete way than here possible, is given by Becker \& Trümper (1999).
} 
RX J1824.5-2452Eis a pulsar-powered synchrotron nebula, similar to what is seen for the Crab. An alternative and maybe more likely interpretation, however, is that RX J1824.5-2452Eis made of a number of point sources, e.g., accreting binaries containing white dwarfs (cataclysmic variables, CV) or neutron stars (low-mass X-ray binaries, LMXB) which could not be spatially resolved by the HRI. An interpretation in terms of low accretion LMXBs is also supported by a long-term X-ray luminosity study of M28. Becker \& Trümper (1999) recently found that the ROSAT PSPC and HRI data taken in 1991 and 1995 suggest an X-ray flux variability of M28 on time scales of years. The total ROSAT HRI energy flux taken in March 1995 from M28 is about a factor of 3 higher than the total energy flux deduced from the March 1991 PSPC observation. This behavior is in line with the results recently published by Gotthelf \& Kulkarni (1997), who discovered an X-ray burst from M28 in the 1995 ASCA data.

- A recent result based on ASCA observations is the detection of X-ray pulses from PSR 1937+21 (Takahashi et al. 1998), a $1.56 \mathrm{~ms}$ pulsar which is the fastest spinning pulsar known so far. Most of the flux (probably up to $80 \%$ ) is in the $1.7-6.5 \mathrm{keV}$ band. The detection is remarkable because the X-ray pulse has a peak width of only $\lesssim 130 \mu$ s (i.e., $\lesssim 0.08$ in phase) and a pulsed fraction of $\sim 80 \%$. The narrow peak implies a very small beaming factor which is not in agreement with a thermal origin of the emission. A nonthermal origin is further suggested by the high pulsed fraction and the spectral analysis. The latter, although strongly limited by photon statistics (less than 100 cts are recorded with the GIS detector), yields a photon-index of $0.9 \pm 1$.

- X-ray pulses from the 2.32-ms pulsar PSR J0218+3242 have been detected by Kuiper et al. (1998) in ROSAT HRI data and, subsequently, with $B e p$ poSAX by Mineo et al. (2000). The BeppoSAX data are best fitted by a power-law spectrum with photon-index $\sim 1 \pm 0.2$ (phase averaged). The pulse profile shows two peaks which change their relative intensity with increasing photon energy. The flux in the energy band $2-10 \mathrm{keV}$ is $f_{x}=4 \times 10^{-13} \mathrm{erg}^{-1} \mathrm{~s}^{-1} \mathrm{~cm}^{2}$ (Mineo et al. 2000). The ROSAT data of PSR J0218+3242 have revealed the existence of a compact synchrotron nebula surrounding the pulsar. The HRI data imply a source extent of about 14 arcsec. Although close to the HRI's attitude solution, the BeppoSAX data seem to confirm the existence of a soft nebula component, showing an energy-dependent pulsed fraction of $37 \pm 13 \%(0.1-2.4 \mathrm{keV}), 67 \pm 12 \%$ (1.6-4 keV), and $89 \pm 10 \%(4-10 \mathrm{keV}$ ) (see Figure 3). A summary of spectral fits based on the BeppoSAX data and the pulse profile for different energy bands is shown in Figure 3.

Noting the spatial coincidence of PSR J0218+4232 with the EGRET source 2EG J0220+4228, Verbunt et al. (1996) tentatively identified the pulsar with the high-energy $\gamma$-ray source. Using some additional EGRET observations, and applying a combination of spatial and timing analysis, Kuiper et al. (1999) conclude that 2EG J0220+4228 is probably multiple: between 0.1 and $1 \mathrm{GeV}$, PSR J0218+4232 is the most likely counterpart, and, above $1 \mathrm{GeV}$, the bright AGN $3 \mathrm{C} 66 \mathrm{~A}$ is the best candidate counter- 

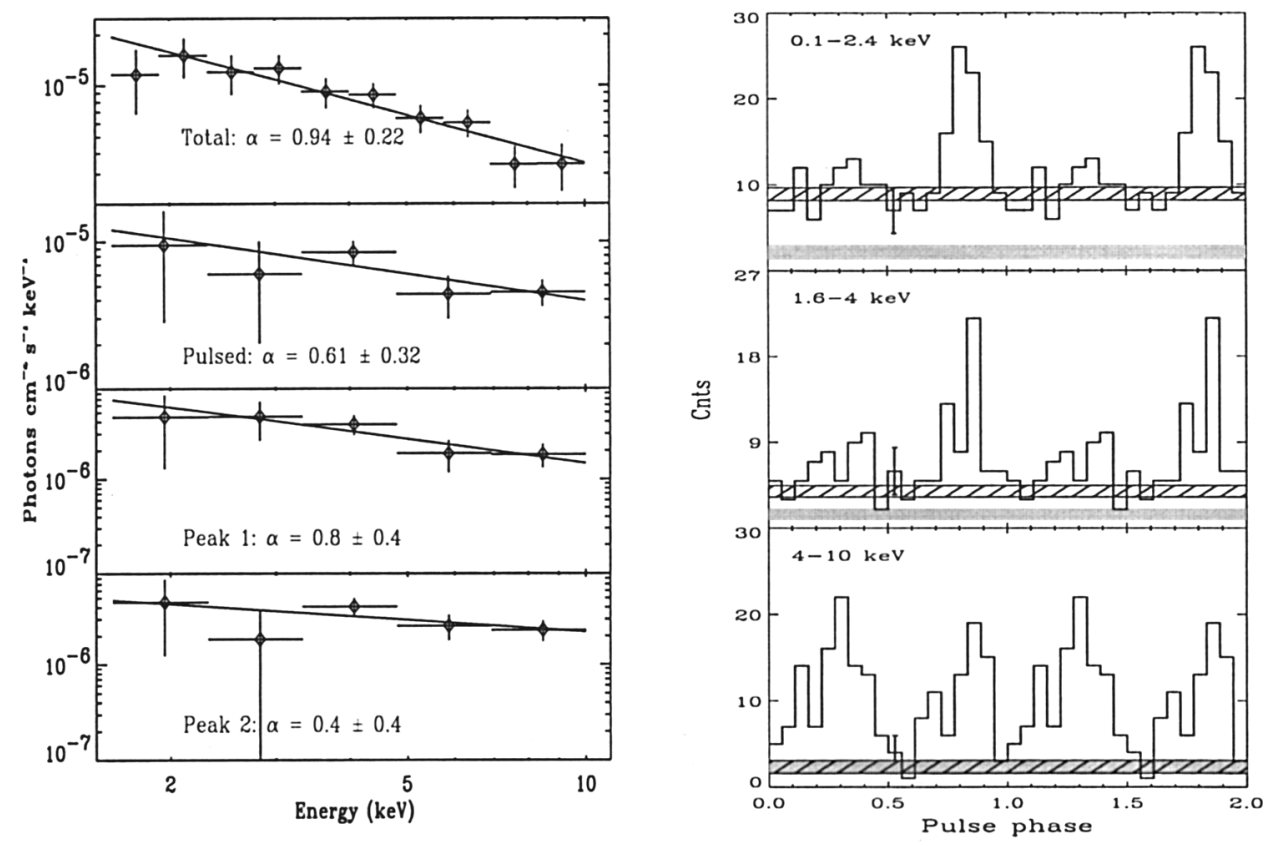

Figure 3. Left: Spectral fits based on the BeppoSAX data of PSR J0218+3242. The upper top panel shows the phase-averaged data fitted with a power-law spectrum (best fit). Fits to the total pulsed emission and to the two peaks seen in the pulse profile are shown in the other panels. Although the small photon-statistics result in large uncertainties, the data suggest a spectral hardening of the pulsed emission with increasing energy. Right: Pulse profiles as observed by ROSAT $(0.1-2.4 \mathrm{keV})$ and BeppoSAX $(1.8-4 \mathrm{keV}$, 4-10 keV). While, in the soft band, only one peak dominates the profile, a second peak appears in the hard band. The gray-shaded area indicates the background level, whereas the hatched area indicates the DC-level. The difference between both levels and its decrease with increasing photon energy support the idea that there is a compact nebula contribution in the soft X-ray band.

part. The third EGRET catalog (Hartman et al. 1999), which is based on more viewing periods than the 2EG catalog, identifies 3EG J0222+4253 (2EG J0220+4228) with $3 \mathrm{C} 66 \mathrm{~A}$, rather than with the ms-pulsar. However, in a note on this source, Hartman et al. (1999) indicate that the identification with $3 \mathrm{C} 66 \mathrm{~A}$ stems from the catalog position based on the $>1 \mathrm{GeV}$ map. Furthermore, they confirm that for lower energies (100$300 \mathrm{MeV}$ ) the EGRET map is consistent with all of the source flux coming from the pulsar, $3 \mathrm{C} 66 \mathrm{~A}$ being statistically excluded.

- The situation for the 5.75-ms pulsar PSR J0437-4715 and the 4.93-ms pulsar 2124-3358 is not as clear as for $1821-24,1937+21$, and J0218+3242, for which a nonthermal origin of the radiation is constrained.

For PSR J2124-3358, there is indication of a double pulse structure in the pulse profile (Becker \& Trümper 1999) which, in terms of pulsed components and pulse phase separation, implies a similarity between the X-ray 
and radio profile observed at $436 \mathrm{MHz}$, possibly pointing towards a nonthermal origin of the X-ray emission. However, the shape of a pulse profile itself is not a strong indicator for the origin of the detected emission if the profile is broad: a radiation cone which yields sharp peaks at one aspect angle may well be seen as barely modulated away from this angle, so that sharp peaks may indicate nonthermal emission processes, whereas the reverse - soft modulated emission originating from thermal processesmust not be true.

The origin of the X-rays detected from PSR J0437-4715 is not yet known. Although PSR J0437-4715 is the brightest of all known X-ray ms-pulsars detected so far, the small bandwidth of ROSAT and the limited photonstatistics of the ASCA and SAX data do not allow discrimination between several possible emission scenarios. Multicomponent thermal spectra (thermal polar-cap emission) and nonthermal spectra (power-law and broken power-law) fit the data equally well.

\section{Summary}

The current findings of pulsar emission properties show that young pulsars with ages of less than $\sim 2000$ years appear Crab-like (i.e., bright synchrotron nebula, sharp X-ray pulses with high pulsed fraction), whereas $\sim 10^{4}-10^{5}$ year old pulsars resemble more the emission properties observed for the Vela pulsar (i.e., $\mathrm{X}$-ray emission beyond $0.5 \mathrm{keV}$ dominated by the emission from the pulsar-driven synchrotron nebula, soft pulses only observable from the Vela-pulsar). However, although these emission properties are found to fit very well for the rotationpowered pulsars (i.e., the radio pulsars), it is quite evident that they are not representative for the whole sample of young neutron stars which exist in our Galaxy. Topics which are discussed in the literature with respect to this are soft gamma-ray repeaters and anomalous X-ray pulsars (see Mereghetti 1999 for a recent review).

The middle-aged pulsars (i.e., the cooling neutron stars) show emission properties which are found to be described by a dichotomy (i.e., two spectral components, soft component is of thermal origin and in agreement with being a relic of the stars heat content during its creation, hard component of nonthermal origin released from within the corotating magnetosphere, transition point at about $0.5-0.6 \mathrm{keV}$ ). Pulsar wind nebulae, as they have been proposed to exist by ASCA observations, are found to be unresolved and unrelated background objects (Becker et al. 1999).

The X-ray emission observed from millisecond pulsars is likely to be dominated by nonthermal processes. This, at least, is observed for PSR 1821-24, PSR 1937+21, and PSR J0218+4232, for which power-law spectra have been measured by ASCA, RXTE, and SAX. For the other millisecond pulsars, the existing data do not allow unambiguous identification of the emission process. Two of the eleven detected millisecond pulsars are found to show diffuse, extended X-ray emission (PSR 1821-24 in M28 and PSR J0218+4232), whereas, for PSR 1821-24, the extended emission possibly comes from unresolved globular cluster sources. The faint, diffuse emission found around PSR J0218+4232 might be plerionic. 
Putting the observed emission properties of the detected pulsars in a somewhat wider frame, Becker \& Trümper (1997) found that the X-ray luminosity of the detected millisecond pulsars show the same linear relationship with the same X-ray efficiency as the Crab-like pulsars (see Figure 4), indicating that the bulk of their emission is mainly due to nonthermal processes as it is for the Crab-like pulsars.

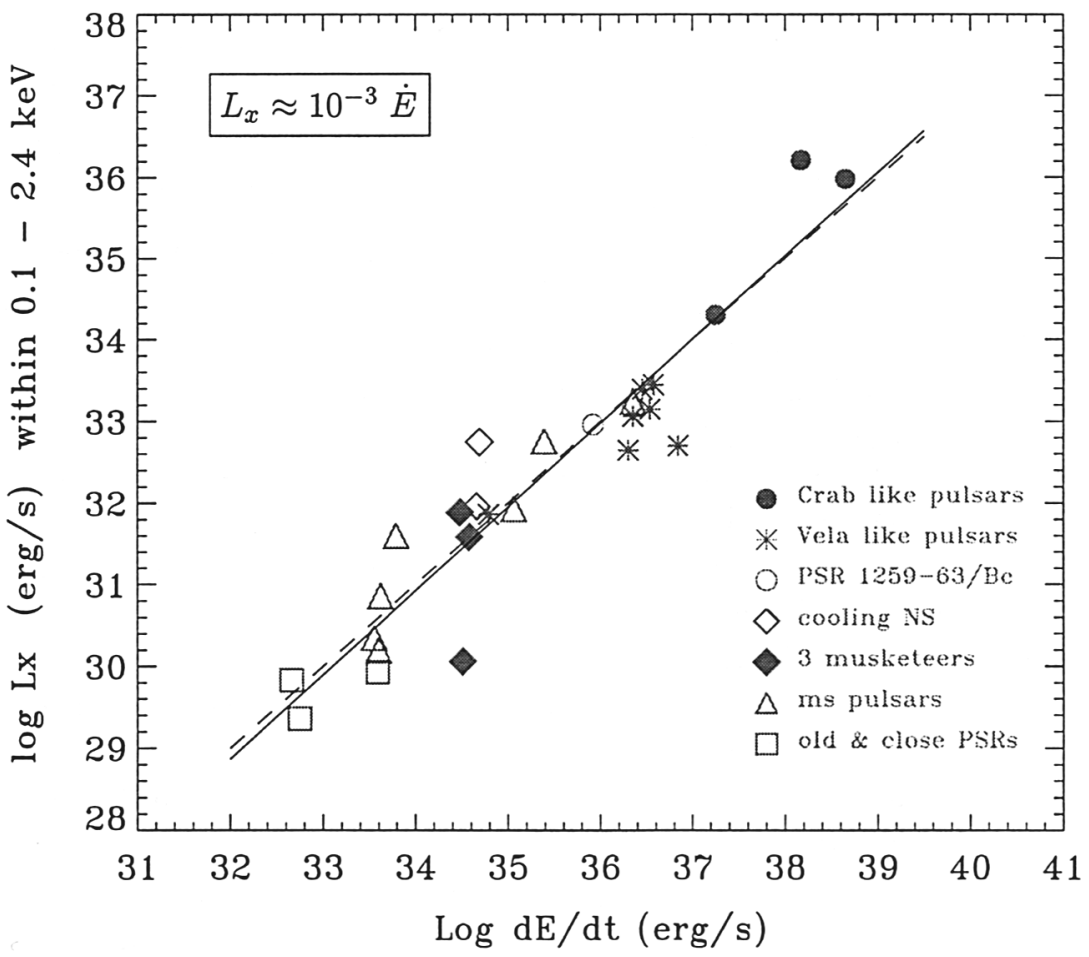

Figure 4. X-ray luminosities of the ROSAT-detected rotation-powered pulsars vs. the pulsars' spin-down energy $\dot{E}$. Thermal spectral components have been subtracted. Although field pulsars and millisecond pulsars form well-separated populations, they obey the same X-ray efficiency (cf. Becker \& Trümper 1997 and discussion therein). The close correlation between $L_{x}$ and $\dot{E}$ strongly suggests that the bulk of the observed X-rays are emitted at the expense of rotational energy, as it is observed for the radio and gamma-ray emission.

\section{References}

Becker, W., \& Aschenbach, B. 1995, in The Lives of Neutron Stars, eds. A. Alpar, U. Kilizóglu, \& J. van Paradijs (Dordrecht: Kluwer), 47

Becker, W., Kawai, N., Brinkmann, W., \& Mignani, R. 1999, A\&A, 352, 532

Becker, W., \& Trümper, J. 1996, A\&AS, 120, C69

Becker, W., \& Trümper, J. 1997, A\&A, 326, 682 
Becker, W., \& Trümper, J. 1999, A\&A, 341, 803

Brazier, K. T. S., \& Becker, W. 1997, MNRAS, 284335

Greiveldinger, C., \& Aschenbach, B. 1999, ApJ, 510, 305

Greiveldinger, C., et al. 1996, ApJ, 465, L35

Gotthelf, E. V., \& Kulkarni, S. R. 1997, ApJ, 490, L161

Halpern, J. P., \& Wang, F. Y.-H. 1997, ApJ, 477, 905

Hartman, R. C., et al. 1999, ApJS, 123, 79

Kawai, N., \& Saito Y. 1999, in 3rd INTEGRAL Workshop, The Extreme Universe (Sept. 1998, Taormina, Italy), in press

Kawai, N., \& Tamura, K. 1996, in ASP Conf. Ser. Vol. 105, Pulsars: Problems and Progress, eds. S. Johnston, M. A. Walker, \& M. Bailes (San Francisco: ASP), 367

Kuiper, L., Hermsen, W., Verbunt, F., \& Belloni, T. 1998, A\&A, 336, 545.

Kuiper, L., et al. 1999, in 3rd INTEGRAL Workshop, The Extreme Universe (Sept. 1998, Taormina, Italy), in press

Marquart, C. B., \& Ögelman, H. 1999, in Workshop on the Relationship Between Neutron Stars and Supernova Remnants, eds. R. Bandiera, E. Masini, F. Pacini, M. Salvati, \& L. Woltjer, Mem. Soc. Astron. Italy, 69, no. 4, 927

Mereghetti, S. 1999, astro-ph/9911252, preprint

Michel, F. C. 1991, Theory of Neutron Star Magnetospheres (Chicago: University of Chicago Press)

Mineo, T., et al. 2000, A\&A, submitted

Ögelman, H. 1995, The Lives of Neutron Stars, eds. A. Alpar, U. Kilizóglu, \& J. van Paradijs (Dordrecht: Kluwer), 101

Ögelman, H., Finley, J. P., \& Zimmerman, H. U. 1993, Nature, 361, 136

Pavlov, G. G. 2000, these Proceedings

Safi-Harb, S., Ögelman, H., \& Finley, J. P. 1995, ApJ, 439, 722

Saito, Y., Kawai, N., Kamae, T., Shibata, S., Dotani, T., \& Kulkarni, S. R. 1997, ApJ, 477, L37

Seward, F. D., \& Wang, Z. R. 1988, ApJ, 332, 199

Shibata, S., et al. 1997, ApJ, 483, 843

Takahashi, M., Shibata, S., Torii, K., Saito, Y., \& Kawai, N. 1998, IAU Circ. 7030

Tamura, K., Kawai, N., Yoshida, A., \& Brinkmann, W. 1996, PASJ, 48, L33

Tsuruta, S. 1998, Phys. Rep., 292, 1

Ulmer, M. P., et al. 1993, ApJ, 417, 738

Verbunt, F., Kuiper, L., Belloni, T., Johnston, H. M., de Bruyn, A. G., Hermsen, W., \& van der Klis, M. 1996, A\&A, 311, L9

Wang, Z. R., Li, Z. Y., \& Begelman, M. C. 1993, Nature, 364, 127

Wang F., Y.-H., Ruderman, M., Halpern, J. P., \& Zhu, T. 1998, ApJ, 498, 373

Yancopoulos, S., Hamilton, T. T., \& Helfand, D. J. 1994, ApJ, 429, 832 